\title{
TRANSFORMACIÓN Y CAMBIO DEL GOBIERNO LOCAL EN EUROPA: UN ESTUDIO COMPARATIVO
}

\author{
CHANGE AND TRANSFORMATION OF LOCAL GOVERNMENT IN \\ EUROPE: A COMPARATIVE STUDY
}

\author{
Hellmut Wollmann \\ Humboldt Universität, Berlín. Alemania/Germany \\ hellmut.wollmann@,rz.hu-berlin.de \\ Ángel Iglesias \\ Universidad Rey Juan Carlos, Madrid. España/Spain \\ angel.iglesias@urjc.es
}

Recibido/Received: 03/06/2011

Aceptado/Accepted: 04/09/2011

\section{RESUMEN}

En este artículo se analizan, desde una perspectiva comparativa e institucional, aspectos clave de los procesos de cambio en los gobiernos locales de Inglaterra, Suecia, Alemania, Francia Italia y España como representativos de la Europa septentrional, central y mediterránea, que se diferencian en dimensiones importantes tales como la arquitectura intergubernamental y la cultura políticoadministrativa. Aunque se constatan tendencias en direcciones coincidentes, se estudian las diferencias y similitudes en orden a comprobar si existen procesos de convergencia o divergencia. Con esta hipótesis de trabajo el estudio se centra en el cambio institucional y en la modernización administrativa a partir de un conjunto de datos empíricos que se agrupan en variables relevantes para analizar tanto el cambio institucional como la modernización administrativa. Del estudio comparativo se desprende que aunque los impulsos transformadores se dan en todos ellos y se dejan entrever tendencias y respuestas comunes, los resultados son distintos en los países estudiados y están condicionados por dimensiones institucionales.

\section{PALABRAS CLAVE}

Neoinstitucionalismo, Gobierno local, modernización administrativa, relaciones intergubernamentales, financiación local.

\section{SUMARIO}

1. Introducción: El neoinstitucionalismo como marco explicativo de la transformación de los gobiernos locales. 2. El gobierno local en el sistema intergubernamental. 3. Gobiernos subnacionales y reformas territoriales. 4. Las competencias locales. 5. Las reformas institucionales del gobierno local. 6. La modernización de la administración local. 7. El gasto público. 8. Conclusiones. Bibbliografía.

\footnotetext{
ABSTRACT

Using a comparative perspective and starting from the concept of institutionalism, this paper provides a systematic and comprehensive analysis of the dynamics of change in local government in six major European countries (England, Sweden, France, Italy and Spain), as representatives of the western,
} 
central and Mediterranean European countries, with country-specific intergovernmental architecture and political and administrative culture. Our study considers a set of variables related to institutional change and administrative modernisation. Although there are common trends, similarities and differences will be estimated in order to shed some light on the degree of their convergence or divergence. The comparative results across countries suggest that although change is a similar trend, there exist significant cross-country specific differences that underly the importance of institutional dimensions.

\section{KEYWORDS}

Neoinstitutionalismo, Local Government, administrative modernization, intergovernmental relations, local finance.

\section{CONTENTS}

1.Introduction: neo-institutionalism as explanatory framework for the transformation of local governments. 2. Local government in the intergovernmental system. 3. Subnational governments and territorial reform. 4. Local government power. 5. Institutional reforms of local government. 6. Modernization of local government. 7. Local government spending. 8. Conclusions. References and documentaries.

\section{INTRODUCCIÓN: EL NEOINSTITUCIONALISMO COMO MARCO EXPLICATIVO DE LA TRANSFORMACIÓN DE LOS GOBIERNOS LOCALES.}

La arquitectura institucional de los sistemas político-administrativos en Europa implica una redefinición de los estilos de hacer política en términos de la creciente interdependencia entre los niveles nacionales y subnacionales, fomentando el cambio institucional y la modernización de estos últimos y, en particular, en el nivel local, dadas sus especificidades (Alba y Vanaclocha, 1987). En efecto, para alcanzar las metas que en el nuevo sistema de relaciones intergubernamentales les tiene encomendadas y que les dan sentido, los gobiernos locales están abocados a una revitalización permanente en el marco de su configuración institucional y de los factores históricos y estructurales en los que operan (Nohlen, 2006).

En Europa los procesos de modernización en los gobiernos locales se producen de forma simultanea y siguen direcciones y patrones coincidentes, aunque esas dinámicas responden a fuerzas diferentes y obedecen a diversos factores por lo que pueden identificarse diferencias de ritmo o velocidad. Con la finalidad de identificar estos factores diferenciales, esté artículo se detendrá en el análisis comparado de la modernización del gobierno local seis países europeos: Inglaterra, Francia, Suecia, Alemania, Italia y España. La elección de estos países está motivada por la intención de incluir países que se distingan lo más posible en rasgos importantes y diferenciados (por ejemplo en su arquitectura intergubernamental y su tradición institucional cultura político-administrativa, entre otros), a fin de identificar las líneas de desarrollo y los factores que las influyen.

Ningún marco teórico es capaz por sí sólo de analizar y explicar el haz de variables históricas, políticas, económicas y sociales que inducen los procesos de modernización en la esfera local. De manera que diferentes aproximaciones teóricas han desarrollado sus propios campos conceptuales para el análisis de las reformas administrativas. No obstante, sin detenernos en un tratamiento pormenorizado, nos apoyaremos en dos marcos explicativos que pueden ayudar a comprender algunos aspectos importantes del fenómeno modernizador. 
En primer lugar y con el objetivo de explicar el desarrollo y cambio institucional (institution building, institutional change) nuestro análisis estará guiado por un marco de estudio que conceptualmente se nutre del llamado neoinstitucionalismo. Las teorías neoinstitucionales esclarecen las causas del cambio en un determinado subsistema políticoadministrativo y las repercusiones generales en el conjunto del sistema. Estas teorías ponen en primer plano la importancia de las estructuras de gobierno como instancias influyentes en el comportamiento y, por lo tanto, en las decisiones de los actores individuales. En su intento de explicar los procesos políticos, el enfoque neoinstitucional pretende aunar en un mismo análisis los factores institucionales y el cambio político y parte de la premisa de que la estructura institucional fija las reglas de juego a las que deberán atenerse los agentes del sistema político (March y Olsen, 1984, 1989; Hall y Taylor, 1996, Kato, 1996, Powell y Dimaggio, Moe, 2005, Peters, 2003, 2006, Thelen, 1999, entre otros).

A efectos del presente estudio, Peters identifica siete tipos neoinstitucionales (Peters, 1999) cuyos rasgos se subsumen en tres variantes entre las que se encuentran el neoinstitucionalismo sociológico, económico e histórico. Otros autores identifican también el institucionalismo económico (North, 1992) y el institucionalismo enfocado en actores, akteurzentrierter Institutionalismus, (Scharpf, 2000). Las transformaciones y cambios en las instituciones se producen a raíz de la evolución de un complejo de factores entre los cuales la cultura política, las estructuras político-administrativas propias de de cada país y el comportamiento de los actores determinan los ajustes institucionales que en cada caso se producen.

Entre las variantes del neoinstitucionalismo, el "neoinstitucionalimo histórico" (historical neoinstitutionalism) hace hincapié en la tradición institucional, cultural etc. y en el impacto que tales herencias institucionales tienen sobre el desarrollo de esas instituciones. Se habla de dependencia de la senda (path dependency) para señalar y captar la influencia del pasado sobre el futuro institucional.

El neoinstitucionalismo histórico sostiene que las instituciones están enraizadas en el devenir de la historia y su tradición o eticidad (Sittlichkeit) lo que impide que se produzcan cambios bruscos. Normalmente, en el marco de las instituciones, los actores cooperan o entran en conflicto al objeto de llegar a acuerdos tácitos ante los problemas que se les plantean y, así, dar respuesta al cambio (un análisis clásico del institucionalismo histórico en True et al., 1999 y Skocpol, 1985). Es probable que los actores tengan opiniones distintas acerca de los problemas $\mathrm{y}$, en la medida que están abocados a llegar a acuerdos, los cambios se producen siguiendo dinámicas incrementales resultado de los procesos de aprendizaje, fomentando más la estabilidad que el cambio (Hall, 1993). Del análisis de la influencia de la de la variable institucional se desprende una consecuencia programática importante: La estabilidad prima por encima de los cambios que se acumulan a partir de ajustes específicos para dar respuestas a cambios en el entorno o contextuales. Los datos disponibles revelan que así ocurre en la mayor parte de países occidentales con sistemas democráticos consolidados (Peters, 1996). Así, los valores y prácticas institucionales enraizados en las organizaciones influyen en el comportamiento de los actores que, por otra parte, cuentan con diferentes grados de poder en su interacción con las organizaciones, de manera que el cambio institucional vendrá determinado por su interacción y la ruta de la senda.

En contraste con la importancia que el institucionalismo histórico otorga a la dependencia de la senda, el institucionalismo económico considera a los actores guiándose por una racionalidad económica en la que prima el utilitarismo y la maximización de su beneficio. De manera que, según este enfoque, las instituciones subordinan su porvenir a las elecciones colectivas que resuelvan las diferencias entre las preferencias individuales de los actores. En consecuencia, lo 
que propone este enfoque es un calco de las propuestas de los teóricos de la Elección Racional que persigue explicar las conductas de elección en las organizaciones políticas y en las estructuras administrativas con la metodología propia de la microeconomía en la que, como presupuesto de su análisis, se encuentra un actor racional que busca maximizar sus utilidades individuales en función de sus preferencias (Downs, 1964, Niskanen, 1971; Tullock, 1965; Olson, 1985).

Adicionalmente, el "institucionalismo relacionado con los actores" (actors-related institutionalism) hace resaltar la importancia de los actores políticos, económicos etc. y de sus intereses, su voluntad etc. como factores cruciales en procesos de gobernanza como resultado de la interacción de actores para regular sus intereses y resolver los problemas derivados de la complejidad, la diversidad y el dinamismo. El concepto incluye los comportamientos informales de actores situados en estructuras policéntricas de toma de decisiones políticas en las que confluyen actores públicos y privados, con intereses contrapuestos en muchas ocasiones y cuyas dinámicas se enmarcan en procesos sociales, políticos y económicos más amplios (Kooimann, 1993). Los actores claves pueden estar ubicados tanto en la arena nacional (o subnacional y local) como en el contexto internacional o supranacional (para lo que la Unión Europea y su Comisión Europea son ejemplares). En su interacción con las instituciones los actores políticos tratan de introducir los cambios más favorables a sus intereses en el marco de las condiciones establecidas por las propias instituciones que les señalan su comportamiento y de su capacidad de influencia.

Bajo estas premisas, el presenta artículo se detiene en el estudio de las variables diferenciales y contextuales (Grotz, 2000:77) que explican los procesos de ajustes institucionales para la configuración de los gobiernos locales. Como punto de partida, dada por supuesta la hipótesis de que existen diferencias notables entre los países estudiados en cuanto a sus respectivas condiciones iniciales de carácter, social, político y económico y reconociendo, además, que dichos países han pasado por etapas de institucionalización anteriores a la configuración actual, se trata de averiguar hasta que punto su desarrollo institucional en lo que afecta a la esfera local se acercan a niveles convergentes o si, por el contrario, persiste y/o se incrementa el alejamiento o divergencia. Para ello nos valdremos del estudio empírico de algunas dimensiones cruciales y analizaremos datos de carácter político, territorial, administrativo y financiero, entre otras.

\section{EL GOBIERNO LOCAL EN EL SISTEMA INTERGUBERNAMENTAL}

El desarrollo histórico de los gobiernos locales se ha debatido entre el centralismo y la autonomía; el predominio del primero ha significado que hasta época reciente los gobiernos locales se identificasen sobre todo con la gestión de los servicios públicos locales. Esto se da en un marco creciente de municipalización del Estado, entendida ésta como la repolitización de la esfera local a consecuencia de los procesos de descentralización que implican que la dependencia entre niveles de gobierno esté alcanzando niveles sin precedentes. La mayor parte de los análisis sobre los pros y los contras de la descentralización ponen de manifiesto que, hasta ahora, sus ventajas han sido mayores que sus inconvenientes (Alba, 1997; Brugué y Gomá, 1998).

A la luz de lo que se termina de decir y habida cuenta de presiones contextuales, entre las que hay que incluir la creciente competencia entre regiones y entre ciudades, los Estados de 
los países europeos han experimentado procesos de centrifugación del poder que se ponen en evidencia con el refuerzo de los niveles subnacionales, fortaleciendo las regionales o, incluso, transformando el Estado unitario o regional en estructuras federales o cuasi-federales con un elevado grados de autonomía y, por tanto, capacidad de negociación con las instancias centrales. El cuadro 1, línea 1, ilustra claramente este fenómeno.

Tal es el caso de Alemania que, con una población aproximada de 82 millones de habitantes, presenta el ejemplo evidente de un país con una tradición histórica e institucional de carácter federal en donde los gobiernos locales tienen un importante papel en el modelo de "federalismo cooperativo" y para la implantación de políticas públicas diseñadas desde el Bund o desde los Estados federados. De igual modo, otro país que tradicionalmente ha mantenido estructuras federales es Suecia, con una población de 9 millones de habitantes que se distribuye en un territorio de tamaño similar al de Alemania. Al igual que en el Reino Unido, la función encomendada a los gobiernos locales ha sido su instrumentalización como prestadores de servicios del Estado de Bienestar, lo que implica una constante relación con el centro.

Por otro lado, la mayoría de los países de la Europa del sur han seguido una tradición unitaria, formando parte de la "familia" napoleónica en la que los niveles subnacionales vienen a ser instancias de representación y acción en el territorio de los poderes centrales. Así, el primer país de este grupo que se desvió de la tradición napoleónica fue España (con 43 millones de habitantes), ya que a partir de la caída del régimen franquista y el restablecimiento del régimen democrático y constitucional, se creó una estructura inter-gubernamental con 17 comunidades autónomas a las cuales se les asignó importantes poderes legislativos y de gestión.

Si hay algo en lo que parecen existir una amplia coincidencia, a la luz de la fuerte posición intergubernamental de las comunidades autónomas en el nuevo esquema constitucional, y aunque existen diversas opiniones respecto a adoptar un término u otro, bien puede calificarse de un sistema cuasi-federal.

Cuadro 1. La Estructura Territorial

\begin{tabular}{|c|c|c|c|c|c|c|}
\hline & Alemania & Francia & R. Unido & Suecia & Italia & España \\
\hline $\begin{array}{c}\text { Federal, } \\
\text { Cuasi-federal } \\
\text { Regional }\end{array}$ & $\begin{array}{l}16 \text { Estados (Länder), } \\
\text { entre ellos 3,„Ciudades } \\
\text { Estado“(Stadtstaaten) }\end{array}$ & & \begin{tabular}{|c|}
2 regiones \\
(Escocia, \\
Gales)
\end{tabular} & & $\begin{array}{c}20 \\
\text { „Regioni““ }\end{array}$ & $\begin{array}{l}17 \text { Comunidades } \\
\text { Autónomas }\end{array}$ \\
\hline Municipal & $\mid \begin{array}{c}323 \text { comarcas (Kreise) } \\
12.196 \text { municipios } \\
(\text { Gemeinden }) \\
116 \text { (Kreisfreie Städte) }\end{array}$ & \begin{tabular}{|} 
21 Régions \\
96 \\
Départements \\
36.569 \\
Communes
\end{tabular} & \begin{tabular}{|c|}
34 \\
counties \\
238 \\
districts/ \\
boroughs \\
83 unitary \\
authorities
\end{tabular} & \begin{tabular}{|c|}
20 \\
comarcas \\
(landstings \\
kommuner) \\
290 \\
municipios \\
(kommuner $)$
\end{tabular} & $\begin{array}{c}103 \\
\text { province } \\
8.101 \\
\text { comuni }\end{array}$ & $\begin{array}{c}50 \text { provincias } \\
8.111 \text { municipios }\end{array}$ \\
\hline $\begin{array}{c}\text { Tamaño } \\
\text { medio de los } \\
\text { municipios } \\
\text { Habitantes }\end{array}$ & $\begin{array}{c}6.690 \\
\text { Land Nordrhein- } \\
\text { Westfalen: } 45.000 \\
\text { Renania-Palatinado } \\
1700 \\
\end{array}$ & 1.560 & $\left|\begin{array}{c}140.000 \\
(\text { districts/ } \\
\text { boroughs })\end{array}\right|$ & 31.300 & 7.270 & 5.430 \\
\hline $\begin{array}{c}\text { Inter- } \\
\text { municipal }\end{array}$ & $\begin{array}{c}1.708 \text { Ämter, } \\
\text { Verwaltungs- } \\
\text { gemeinschaften,etc. }\end{array}$ & \begin{tabular}{|c|}
12.840 \\
Syndicats \\
\\
2.601 \\
Communautés
\end{tabular} & & & \begin{tabular}{|c|}
356 \\
Comunità \\
montane \\
278 Unioni \\
di comuni
\end{tabular} & $\begin{array}{c}81 \text { Comarcas } \\
\text { ca. } 1.000 \\
\text { Mancomunidades } \\
\text { de municipios }\end{array}$ \\
\hline
\end{tabular}

Fuente: Elaboración propia a partir de datos proporcionados por Dexia $(2006,2008)$ 
Y algo similar sucede en Italia que, con 58 de millones habitantes, se ha alejado también de la tradición napoleónica en el transcurso de los años 90 del pasado siglo, cuando significativas competencias legislativas y de gestión fueron transferidas del Estado central a las 20 regiones. Asimismo se habla de una forma de cuasi-federalismo.

Por el contrario, Francia, con 60 millones de habitantes, presenta una situación ambigua. Por un lado ya en 1982 Francia puso en marcha un proceso de descentralización ambicioso, tratando de romper son con la arquitectura napoleónica unitaria y centralizada. Entre otras medidas se establecieron 21 regiones (régions) con Parlamentos electos en el nivel regional. Al mismo tiempo, se decidió, plasmándolo así constitucionalmente, que las regiones poseyeran el mismo estatus institucional que los departamentos y los municipios, es decir, que los dos niveles del gobierno local ya existentes, conformándose así un tercer nivel del sistema del gobierno local (collectivités locales). De manera que explícitamente se excluyó el que las regiones mantuvieran un estatus jurídico-político superior a los dos niveles que existían con anterioridad.

Ahora bien, para mostrar la importancia de los municipios dentro de la estructura intergubernamental, en una perspectiva comparada, es preciso detenerse en los porcentajes de los empleados públicos por niveles, pues ello da idea de las funciones y competencias encomendadas a los municipios en términos de gasto público. En el cuadro 2 se resumen estos datos.

Cuadro 2. Porcentaje de Empleo Público

\begin{tabular}{|c|c|c|c|c|}
\hline & Local & Regional (Estados) & Estatal & Agencias Públicas \\
\hline Alemania & $35 \%$ & $53 \%$ & Bund $12 \%$ & \\
\hline Francia & $30 \%$ & & $51 \%$ & $\begin{array}{c}\text { Hospitales publicos } \\
19,0 \%\end{array}$ \\
\hline Reino Unido & $56 \%$ & & $16,9 \%$ & National Health Service $26 \%$ \\
\hline Suecia & $83 \%$ & & $17 \%$ & \\
\hline Italia & $13,6 \%$ & $3,8 \%$ & $54,7 \%$ & $\begin{array}{c}\text { Servizio Sanitario Nazionale } \\
20,3 \%\end{array}$ \\
\hline España & $23,6 \%$ & $49,9 \%$ & $22,5 \%$ & \\
\hline
\end{tabular}

Fuente: Elaboración propia a partir de datos proporcionados por Dexia (2008)

Estos datos revelan que, respecto a Alemania y España como países federales y cuasifederales respectivamente hay que destacar que sus niveles regionales, es decir los Länder y las comunidades, emplean aproximadamente la mitad del conjunto de los ocupados públicos. Resalta una diferencia entre los dos países en el porcentaje de los empleados del nivel central y del nivel local. En Alemania los empleados federales constituyen solamente el 10 por ciento lo que refleja la disposición constitucional que al gobierno federal no es permitido establecer unidades administrativas propias en el espacio subnacional. Al mismo tiempo, el porcentaje de los empleados del gobierno local asciende al 35 por ciento en Alemania - en contraestación al 23.6 por ciento en España. Eso indica que el peso funcional des sector local resulta mayor en Alemania que en España.

En este contexto conviene hacer notar que en Francia, no obstante los avances en la descentralización desde la década de los ochenta del pasado siglo, el porcentaje de empleo público de la administración central aún asciende a más del cincuenta por ciento del total 
sector público. Esto revela, entre otras cosas, que, aunque formalmente, las políticas de descentralización no han contemplado el traspaso material de competencias a los niveles subnacionales y, particularmente, al nivel local. Con todo, persisten solapamientos tanto en las estructuras administrativas como en la función pública que ponen de la herencia del modelo napoleónico y su "dependencia de sendero".

A este respecto y como contraste, merece la pena destacar el caso de Suecia donde el personal del Estado representa sólo el 17 por ciento del conjunto de empleados públicos, mientras que el personal de los dos niveles del gobierno subnacional llega al ochenta tres por ciento. Eso demuestra el grado excepcional de descentralización de este país donde las funciones y tareas consustanciales al Estado de Bienestar son realizadas por los gobiernos locales.

Para concluir este apartado es preciso hacer referencia a dos cuestiones que ilustran los casos de España y Alemania como ejemplos típicos de los procesos de federalización. En primer lugar y puesto que los niveles regionales, bien los Länder en Alemania o las Comunidades Autónomas en España disponen de competencia exclusiva para legislar sobre materias importantes, surgen tensiones entre los principios del Estado unitario que exige la igualdad para todos los ciudadanos en el conjunto del territorio del Estado y la potestad legislativa ejercida por cada una de las regiones en fijar sus propias regulaciones y actos normativos. Esta situación aboca a que puedan originarse disparidades y distorsiones sociales y económicas entre las regiones que van en contra de disposiciones fundamentales de la Constitución y que acarrean importantes conflictos de carácter político y jurisdiccional.

En segundo lugar, el federalismo o cuasi-federalismo puede debilitar la posición del nivel local en el sistema intergubernamental puesto, como también ilustran los casos de España y Alemania, las regiones se comportan, en su relación con el Estado, como celosos valedores de la descentralización, mientras, por el contrario, en su relación con el nivel local, actúan con verdadero furor neocentralista en su afán de controlar sus propios niveles locales. Así, los gobiernos locales pueden encontrarse, en un sistema federal o cuasi-federal, en una posición jerárquicamente más dependiente que tal vez en un país unitario lo que, a primera vista, parece algo paradójico (Wollmann/ Bouckart 2006: 23).

\section{GOBIERNOS SUBNACIONALES Y REFORMAS TERRITORIALES}

Como las líneas 2 y 3 del Cuadro 1 ilustran, existen considerables diferencias entre los países en cuanto al tamaño medio de los municipios (es decir, de las unidades subnacionales del nivel inferior). Con respecto a los seis países que aquí estamos comparando, el tamaño medio varía entre 140.000 habitantes en Inglaterra y 1.560 en Francia. Dicho de otra manera, pueden distinguirse dos grupos de países: a) Por un lado, los países con municipios de tamaño medio elevado. A la cabeza de este grupo figura Inglaterra con districts/boroughs que, por término medio, cuentan con 140.000 habitantes. En el caso de Suecia el tamaño medio asciende a 31.000 habitantes. En este grupo, en los años sesenta y setenta del siglo pasado, se iniciaron e implantaron reformas territoriales de los municipios a través de fusiones e integraciones territoriales de los pequeños municipios y localidades. En las estrategias perseguidas por los gobiernos centrales de estos países se establecieron comisiones de carácter tecnocrático para preparar los proyectos de reforma que, posteriormente, eran sometidas a debate público con los municipios afectados y los ciudadanos con la finalidad de llegar a acuerdos acerca de la estructura territorial definitiva. Pero lo cierto es que en los casos en que se produjeron protestas 
o incluso una oposición frontal a las propuestas tecnocráticas, los parlamentos nacionales saldaron cualquier discusión y, a la postre, decidieron la estructura territorial de una manera definitiva y, allí donde lo consideraron necesario, coercitiva, legitimándose en la convicción de cultura política de que el parlamento posee la potestad de decidir y fijar los confines y el tamaño de los municipios. En la literatura comparativa se habla del "modelo noreuropeo".

De otro lado, pueden identificarse países en los que tradicionalmente ha existido una estructura territorial muy fragmentada con pequeños municipios y localidades. El ejemplo clásico es Francia con aproximadamente 36.500 municipios (communes) que, por término medio, cuentan con 1.560 habitantes. Los términos municipales de estos municipios se establecieron de la Revolución Francesa de 1789 que vino a confirmar el inframunicipalismo histórico francés y, prácticamente la planta municipal ha permanecido así desde entonces.

Ni que decir tiene que España se incluye también en este grupo. Aunque no llega al grado de dispersión francés, el inframunicipalismo es también una característica definitoria de la planta municipal española con una media de 5.400 habitantes por municipio. Tanto en Francia como en España no se han diseñado reformas territoriales tendentes a reducir la planta municipal en el sentido de aumentar el tamaño de los municipios. Aunque se reconoce que el aumento del tamaño incidiría en una mejora prestación de servicios públicos, las estrategias elegidas se sustentan en el principio de voluntariedad según el cual se permite la fusión e integración de los municipios siempre y cuando exista un acuerdo del Pleno y sea ratificado por los vecinos. Este es el modelo que en la literatura comparada se conoce como el modelo de Europa del Sur (Norton 1994: 40).

En el caso de Francia, al rasgo de voluntariedad, enraizado en su cultura política, se añade otra variable focal, cual es la capacidad de influencia de los alcaldes (maires) en el Parlamento Nacional (Assemblée Nationale) y que tiene su razón de ser en el sistema del "cumul de mandats", a partir del cual un importante número de alcaldes son, al mismo tiempo, miembros del Pleno municipal y diputados en el Parlamento Nacional o en el Senado. Esta situación les otorga una importante capacidad de influencia sobre la política nacional, incluyendo, claro está, las decisiones que en sede parlamentaria se tomen acerca de la estructura territorial local.

En cualquier caso, los países que se encuentran con dificultades para llevar a cabo reformas territoriales mediante la aprobación de legislación nacional han adoptado y fijado esquemas y estructuras institucionales para alentar a la cooperación intermunicipal buscando la eficacia, economía y eficiencia en la prestación de servicios públicos. El ejemplo paradigmático es Francia y su larga tradición intermunicipal. En 1890 se estableció un marco institucional en el que los syndicats debían llegar a acuerdos para institucionalizar la cooperación entre municipios bien para funciones específicas, o para funciones de carácter más generalista. A partir de la década de los sesenta del pasado siglo se incluyó en este marco institucional la figura de la communauté, es decir une especie de unión de municipios, con la finalidad de garantizar formas más integradas de cooperación intermunicipal. En este sentido bien podría afirmarse que la intermunicipalidad es el resultado de largos procesos históricos y es un distintivo clave del sistema político francés. Más recientemente, la Ley Chevènement de 1999 ha introducido tres tipos de communautés para simplificar la complejidad y multiplicidad de la intermunicipalidad. En la actualidad esta abarca casi 13.000 syndicats y aproximadamente 2.600 communautés.

En España, a falta de una reforma de la planta municipal se ha conformado una red de entes intermunicipales con el objetivo de facilitar y fomentar la cooperación intermunicipal 
entre, sobre todo, pequeños municipios en el ámbito de una misma Comunidad Autónoma. En Cataluña, con una arraigada tradición comarcal ha habido intentos de recuperar ese ámbito territorial en detrimento, incluso, de las provincias, pero las comarcas, aunque existen formalmente, apenas tienen competencias materiales. Actualmente, en todo el territorio del Estado Español están reconocidas 81 comarcas y aproximadamente 1.000 mancomunidades de municipios.

Por referirnos ahora al caso alemán, quizás su rasgo distintivo es que persiste una notable variedad en cuanto a las estrategias de reformas territoriales dependiendo de los Länder, puesto que cado uno de ellos ostenta la potestad decisoria sobre la estructura territorial de los municipios radicados en su territorio. Sin embargo, se pueden distinguir dos tipos de Länder. Por un lado, aquellos que decidieron a implantar una reforma a través de la fusión e integración de pequeños municipios y mediante la creación de ciudades de mayor tamaño, dando como resultado un incremento considerable en el tamaño medio de los municipios. En el Land Nordrhein-Westfalen, por ejemplo, el tamaño medio de los municipios asciende a 45.000 habitantes, lo que podría incluirse en el modelo del norte de Europa al que antes hemos hecho referencia.

De otra parte, la mayoría de los Länder, incluyendo los de la antigua Alemania del Este, decidieron, a partir de 1990, mantener el status quo territorial o, excepcionalmente llevar a efecto reformas menores de su planta municipal y a institucionalizar, en lugar de ello, entes intermunicipales para dotar a los pequeños municipios de un aparato administrativo suficiente que les permitiera ser operativos en las redes de cooperación intermunicipal. Estas estrategias se identifican más con el modelo "sur-europeo" y, en cierto modo, toma como modelo lo acaecido en Francia, de manera que, actualmente existen unos 1.700 entes intermunicipales en el conjunto del Estado alemán. Quizás no estaría de más señalar que estas estrategias se han implantado a partir de la legislación aprobada por los Lánder y que ha resultado de obligado cumplimiento por parte de las poblaciones afectadas, más allá de las protestas o las demandas de participación ciudadana que han resultado inatendidas.

Diríamos, en fin, que tanto Alemania como Francia han incluido recientemente en su agenda política la necesidad de potenciar sus sistemas de intermunicipalidad en el sentido de agrupar a un mayor número de municipios y dotar a los entes intermunicipales de órganos de decisión suficientemente representativos para permitir una mejora en la prestación de servicios públicos a los entes locales. Es posible que España siga también esta vía para dotar de mayor capacidad a su sistema local.

Comoquiera que sea, la solución de la intermunicipalidad también presenta disfunciones. Primero, buen número de pequeños municipios están infra-representados en los órganos intermunicipales; es más, en muchos de ellos ni siquiera hay candidatos para las elecciones locales por lo que bien podría afirmarse que, en estas pequeñas localidades la democracia local no encuentra instrumentos para hacerla operativa. Segundo, los entes intermunicipales encuentran enormes dificultades para fomentar y facilitar la cooperación entre sus municipios. Más bien al contrario, parece que los intereses particulares generan numerosos problemas de coordinación, incrementándose así los costes de transacción. Tercero, desde el punto y hora en que los entes municipales carecen de elección directa de sus órganos dirigentes (son elegidos por los Plenos de los municipios que los integran), se generan problemas de legitimidad democrática.

En este contexto, algunos Länder de la antigua Alemania del Este han impulsado recientemente una nueva oleada de reformas territoriales que están dirigida a crear mayores ciudades mediante la fusión e integración de pequeños municipios, reduciendo al mismo 
tiempo el numero de los entes intermunicipales (Wollmann 2009b). De igual manera, en Francia, es decir, en el país de la intermunicipalidad por excelencia, la crítica a los entes intermunicipales está siendo cada vez más fuerte. En octubre de 2008 el Presidente Sarkozy puso en marcha una comisión (Comité Balladur) con el mandato de elaborar recomendaciones acerca del gobierno local pero, sobre todo, de la intermunicipalidad. En febrero de 2009 la comisión publicó su informe (Comité Balladur 2009). Algunas de las recomendaciones claves de esta comisión señalan la importancia de simplificar y reducir la intermunicipalidad y de abrir el sendero legal e institucional hacia el establecimiento de ciudades de mayor tamaño mediante la fusión e integración municipal. Habrá que ver el alcance real de estas recomendaciones y en qué medida llegan a implantarse.

\section{LAS COMPETENCIAS LOCALES}

En lo que hace a las funciones desempeñadas por los gobiernos locales existen notables diferencias así como semejanzas entre los países aquí estudiados. A partir de datos agregados, en el cuadro 3 se ilustra el nivel de participación de los municipios en las políticas públicas y prestación de servicios relacionadas con las prestaciones principales del Estado de Bienestar.

Cuadro 3. Porcentaje de gastos por niveles de gobierno

\begin{tabular}{|c|c|c|c|c|c|c|c|c|c|}
\hline & \multicolumn{3}{|c|}{ Alemania } & Francia & $\begin{array}{c}\text { Reino } \\
\text { Unido }\end{array}$ & Suecia & Italia & \multicolumn{2}{c|}{ España } \\
\cline { 2 - 10 } & Municipios & Comarcas & Länder & $\begin{array}{c}\text { los tres } \\
\text { niveles }\end{array}$ & $\begin{array}{c}\text { los dos } \\
\text { niveles }\end{array}$ & $\begin{array}{c}\text { los dos } \\
\text { niveles }\end{array}$ & $\begin{array}{c}\text { Incluyendo } \\
\text { regioni }\end{array}$ & $\begin{array}{c}\text { Muni- } \\
\text { cipios }\end{array}$ & $\begin{array}{c}\text { Comuni- } \\
\text { dades } \\
\text { Auton. }\end{array}$ \\
\hline $\begin{array}{c}\text { Administra- } \\
\text { ción } \\
\text { General }\end{array}$ & $12,7 \%$ & $8,1 \%$ & $33,2 \%$ & $19,2 \%$ & $8,1 \%$ & $10,0 \%$ & $14,6 \%$ & $33,4 \%$ & $9,9 \%$ \\
\hline $\begin{array}{c}\text { Bienestar } \\
\text { Social }\end{array}$ & $26,4 \%$ & $49,1 \%$ & $11,1 \%$ & $15,8 \%$ & $29,0 \%$ & $27,0 \%$ & $4,6 \%$ & $8,3 \%$ & $5,2 \%$ \\
\hline $\begin{array}{c}\text { Orden } \\
\text { Público }\end{array}$ & $6,4 \%$ & $6,3 \%$ & $6,1 \%$ & $2,8 \%$ & $10,0 \%$ & $0,9 \%$ & $1,5 \%$ & $7,9 \%$ & $2,6 \%$ \\
\hline $\begin{array}{c}\text { Cultura y } \\
\text { Deporte }\end{array}$ & $11,3 \%$ & $2,3 \%$ & $2,2 \%$ & $10,2 \%$ & $3,3 \%$ & $3,4 \%$ & $3,0 \%$ & $10,9 \%$ & $3,2 \%$ \\
\hline Educación & $10,1 \%$ & $14,6 \%$ & $30,0 \%$ & $16,2 \%$ & $30,0 \%$ & $21,6 \%$ & $8,3 \%$ & $4,5 \%$ & $28,0 \%$ \\
\hline Salud & $9,0 \%$ & $4,4 \%$ & $1,5 \%$ & $0,6 \%$ & 0 & $27,1 \%$ & $43,0 \%$ & $1,3 \%$ & $35,0 \%$ \\
\hline
\end{tabular}

Fuente: Elaboración propia a partir de datos proporcionados por Dexia (2008)

Del cuadro anterior es preciso destacar:

- En todos países del estudio los municipios desempeñan importantes funciones en materia de asuntos sociales. Esto es sobre todo evidente en los municipios de Suecia, Inglaterra y Alemania.

- Las competencias en materia de cultura una importante función en los gobiernos locales de España, Francia y Alemania.

- Como una competencia figura la prestación de servicios públicos básicos (abastecimiento de agua, tratamiento de aguas residuales, transporte público 
municipal y abastecimiento de energía, entre otros). Esto se observa particularmente en Alemania y en Suecia, pero también in Italia.

- Hay importantes diferencias en las esferas de educación y salud pública.

- En los países continental-europeos la educación obligatoria se considera tradicionalmente como responsabilidad del Estado, sea del Estado central (en los países de tradición unitaria) sea del nivel regional (Länder, Comunidades Autónomas) en países federales o cuasi-federales. En estos últimos los municipios son responsables de los gastos en infraestructuras y mantenimiento. Por el contrario, en Suecia y en Inglaterra los niveles del gobierno local ostentan responsabilidad plena en cuanto a la enseñanza obligatoria se refiere.

- Pero, sobre todo, las grandes divergencias aparecen en el ámbito de las prestaciones sanitarias debido fundamentalmente a las diferencias existentes en los sistemas nacionales de seguro de enfermedad.

De cualquier forma, del peso y la envergadura que las funciones ejercidas por los municipios tienen dentro del sistema intergubernamental puede inferirse del porcentaje de participación del gasto municipal respecto al total del gasto público a que se refiere el cuadro 4. En este aspecto, destaca el enorme peso funcional del gobierno local en Suecia y, por tanto, su elevado grado de descentralización territorial.

Por el contrario, en España y en Alemania el porcentaje de los gastos de los municipios asciende, respectivamente, al 12 y 11.7 por ciento, lo que pone de manifiesto la gran importancia que en estos países adquiere el nivel regional (Comunidad Autónoma o Land) en el sistema intergubernamental.

Cuadro 4. El Gasto Público Municipal

\begin{tabular}{|c|c|c|}
\hline & $\begin{array}{c}\text { Porcentaje de gasto de los municipios } \\
\text { en el total de Gasto Público }\end{array}$ & $\begin{array}{c}\text { Gasto local por habitante } \\
\text { (en euros) }\end{array}$ \\
\hline Alemania & $11,7 \%$ & $1.498 €$ \\
\hline Francia & $8,2 \%$ & $1.211 €$ \\
\hline Reino Unido & $29,5 \%$ (los dos niveles) & $3.930 €($ los dos niveles) \\
\hline Suecia & $27,1 \%$ & $4.923 €$ \\
\hline Italia & $8,8 \%$ & $1.040 €$ \\
\hline España & $12,0 \%$ & $1.000 €$ \\
\hline
\end{tabular}

Fuente: Elaboración propia a partir de datos proporcionados por Dexia (2008)

\section{LAS REFORMAS INSTITUCIONALES DEL GOBIERNO LOCAL}

Por referirnos ahora a las instituciones y al proceso político en la arena local, en la mayoría de los países europeos el sistema del gobierno local ha experimentado importantes cambios, particularmente en dos aspectos nodales (Wollmann 2008c). En primer lugar, en algunos países el tradicionalmente vigente principio de la democracia representativa que se manifiesta en la supremacía del Pleno ha sido complementado por el referéndum local decisorio como mecanismo de la democracia local directa. La introducción del referéndum local puede considerarse como un paso crucial en un avance general orientado al empoderamiento (empowerment) ciudadano al permitirles mayor participación en los asuntos 
que les atañen. Los referidos locales decisorios fueron introducidos en todos los Länder alemanes ya desde principios de la pasada década de los noventa, lo mismo que en Italia. La experiencia en Alemania pone de manifiesto que su introducción ha tenido un impacto importante en la arena e interacción política local, puesto que, en el caso de un conflicto y disenso entre el Pleno y la ciudadanía, los ciudadanos pueden, por medio de un referéndum, superar la voluntad y decisión del Pleno e imponer su voluntad. Se dispone ya de múltiples ejemplos de materias importantes que se deciden vía referéndum, con, a veces, resultados controvertidos.

En segundo lugar, se han llevado a la práctica importantes cambios institucionales en los que subyace un impulso para reformar y reforzar el liderazgo político y administrativo dentro del gobierno local. En este aspecto, se hace preciso, de nuevo, la distinción de dos grupos o tipologías en el esquema del gobierno local. Por un lado, puede señalarse el modelo ingles y escandinavo que se caracterizan por el ejercicio de la función gubernamental por medio de comisiones (government by committee). Históricamente eso ha venido a significar que, por principio, el conjunto de concejales elegidos, como colectividad, decide en todos y cada uno de los asuntos locales y, al mismo tiempo, dirige la administración, de manera que, distintas comisiones, dentro de sus responsabilidades pectorales, ejercen los poderes correspondientes.

Este sistema de gobierno viene siendo criticado por la fragmentación sectorial que supone e, incluso en su falta de transparencia y dación de cuentas. Por ello, tanto en el caso de Inglaterra como en Suecia se han iniciado reformas que, en síntesis, apuntan a transformas este esquema hasta otro en el que prima la parlamentarización del gobierno local con un con un "gabinete", compuesto de concejales del partido que obtenga la representación mayoritaria y su "líder".

Pero, si se pone en paralelo a los países continental-europeos, éstos han seguido una senda institucional en la que ha regido el principio de separación de poderes por el cual el Pleno y los concejales actúan a modo de parlamento local, mientras que la función ejecutiva está desempeñada por el alcalde quién, a su vez es un miembro del Pleno y es elegido por éste.

Más allá de estas diferencias, son dos las razones que se aducen para impulsar el liderazgo político local. La primera hace referencia a las exigencias de profundizar en la democracia local, fomentando la participación ciudadana y que los ciudadanos elijan directamente a los alcaldes. La segunda tiene que ver con las exigencias en cuanto a la obtención de resultados, de tal manera que la elección directa del alcalde se pergeña como una estrategia para fortalecer el liderazgo político y administrativo con vistas a facilitar la gobernanza del municipio. Y son, de nuevo, en este aspecto, Alemania e Italia los países que sirven de ejemplo. En Alemania, ya en los años cincuenta del pasado siglo, dos Länder fueron pioneros en la elección directa del alcalde (Bürgermeister) que, al propio tiempo, es el presidente del Pleno y está a la cabeza de la administración municipal. La combinación del papel político, el ejercicio del poder ejecutivo y la legitimidad que otorga la elección directa, determina una posición presidencialista del alcalde (strong mayor) en la que se viene a configurar un modelo de gobierno local de carácter presidencialista. Desde el inicio de los anos noventa este régimen de posibilidad de elección directa del alcalde fue introducido en todos Länder alemanes. De forma simultanea y con el objeto de ampliar el potencial participativo de los ciudadanos y limitar y controlar la posición del alcalde, en algunos de los Lánder, se posibilitaron procedimientos para destituirle (Abwahl) por medio de un referéndum. Allí donde han sido instituidos, estos procedimientos de destitución del alcalde se han llevado a la 
práctica, por lo que la introducción de la elección directa puede considerarse como una innovación exitosa.

\section{LA MODERNIZACIÓN DE LA ADMINISTRACIÓN LOCAL.}

Preguntándonos ahora por el desarrollo de la modernización de la administración municipal en países europeos, resulta de nuevo pertinente la distinción de varios grupos o tipologías de países y de sus correspondientes tradiciones y cultura administrativa locales (para un estudio comparativo a propósito de este aspecto, puede consultarse el considerado como clásico de Pollitt/Bouckaert 2004, y para un análisis focalizado en Francia y Alemania véase Wollmann/Bouckaert 2006 y Kuhlmann 2009).

A partir de los planteamientos de estos autores pueden distinguirse dos grupos de países: A) El grupo anglosajón con el Reino Unido como paradigma; la característica distintiva de este grupo es la primacía del Common Law que no reconoce una distinción entre derecho público y privado y, por ende, entre sector público y sector privado, de modo que a los empleados públicos de cualquier nivel de gobierno, les son de aplicación las mismas normas laborales que al conjunto de los trabajadores. B) El grupo de países de tradición continentaleuropeo y que se reconocen en los ejemplos de Francia y Alemania; los países pertenecientes a este grupo se entroncan en la tradición del Derecho Romano a partir del cual se estableció claramente la distinción entre derecho público y privado y, en consecuencia, entre sector público y sector privado; de manera que los empleados públicos están sometidos a normas laborales propias; también las administraciones públicas en estos países deben atenerse en su actuación a los principios del Estado de Derecho, según el cual las administraciones públicas están sometidas al principio de legalidad y al control judicial; de manera que sus administraciones públicas mantienen unas estructuras organizativas de carácter jerárquico y actúan, respondiendo a las exigencias del Estado de Derecho, de acuerdo con las disposiciones legales, es decir, se conducen conforme a la racionalidad legal weberiana.

Así las cosas, hace ahora ya tres décadas, se origino en Inglaterra el movimiento de la llamada Nueva Gestión Publica (New Public Management). Con el apoyo del gobierno tatcherista, inspirado por conceptos políticos y económicos neoliberales sustentados en las viejas Teorías de la Elección Racional y en las algo más recientes de la Nueva Economía Institucional, el mensaje de la Nueva Gestión Publica perseguía varios objetivos: Primero, reducir el ámbito de actuación del Estado del Bienestar mediante su adelgazamiento (lean state). Segundo, flexibilizar internamente la administración pública suprimiendo los planteamientos weberianos. Tercero, delegar, comisionar y externalizar (outsourcing) la prestación de servicios públicos a agentes y proveedores externos bien fueran privados, semi públicos o públicos introduciendo mecanismos de mercado y la lógica de la competencia. El núcleo central del programa de la Nueva Gestión Pública era, en definitiva, trasladar al sector público principios de comportamiento y actuación del sector privado.

Lo cierto es que en los países anglosajones de la que era originaria, la Nueva Gestión Publica tuvo un importante impacto en la administración publica. Aparte de presiones políticas, la falta tradicional de una distinción conceptual entre el sector publico y privado contribuyó sobremanera a favorecer la transmisión y adaptación de conceptos e instrumentos del sector privado al sector publico. De manera que buena parte de los servicios de titularidad y competencia pública, tanto del ámbito estatal como de los ámbitos subnacionales, fueron comisionados a prestadores externos, tanto públicos como privados y/o privatizados. 
En los países de la Europa continental y desde los años noventa, la modernización de la administración pública mostraba una trayectoria notablemente distinta de la anglosajona debido a sus diferentes presupuestos institucionales y culturales. En estos países, el concepto tradicional del Estado de Derecho (con su prevalencia de las disposiciones legales) y la persistencia modelo burocrático weberiano han limitado el impacto de los mensajes y recomendaciones de la Nueva Gestión Publica, difundidos desde organizaciones internacionales tales como la OCDE o el Fondo Monetario Internacional, entre otras.

A este respecto, puede de nuevo traerse a colación el caso alemán. A principios de los noventa y por influjo de la Nueva Gestión Publica anglosajona, en realidad mediante un calco de la misma, se diseño el denominado Neues Steuerungsmodel o nuevo modelo de gestión, cuyo objetivo primordial era la flexibilización de las estructuras jerárquicas de las administraciones públicas. Algunas de las transformaciones llevadas a cabo bajo su influjo han propiciado cambios puntuales en la administración pública, especialmente en el nivel municipal. Ahora bien, en el conjunto de las organizaciones públicas su impacto ha sido limitado. Un reciente trabajo de investigación pone de manifiesto que la viabilidad de las propuestas reformadoras descansa en la aplicación de los conceptos reformistas que ya se aplicaron en la década de los setenta que, en algunos casos, se pueden complementar con las propuestas del Neues Steurungsmodel (Kuhlmann et al., 2008). Es decir, lo que en la literatura comparativa se conoce como modelo administrativo neoweberiano" que persigue fusionar aspectos del tradicional modelo weberiano con elementos propios de la Nueva Gestión Publica.

Otro ámbito en el que se han producido cambios en el gobierno local ha sido en la prestación de servicios públicos de carácter básico, muchos de los cuales se incluyen en la categoría de la denominada Daseinsvorsorge o procura existencial, incluyendo el abastecimiento de agua, tratamiento de residuos, transporte público, energía o servicios educativos, entre otros). Tradicionalmente tales prestaciones de servicios se realizaban bajo la fórmula de las Stadtwerke con la creación e intervención de empresas públicas municipales. Las crisis presupuestarias y las presiones liberalizadoras provocaron la venta al sector privado de una gran mayoría de empresas municipales encargadas de la prestación de servicios básicos. Sin embargo, recientemente parece observarse un movimiento pendular mediante el cual los gobiernos locales están recomprando sus empresas, de tal manera que comienza a hablarse de un proceso de remunicipalización (Baldersheim et al., 2010).

Las crisis financieras, que propiciaron el proceso de reformas administrativas, trajeron consigo, en el caso de Alemania, una reducción importante de los empleados públicos municipales (Cuadro 6), algo que no ha ocurrido, al menos al mismo nivel, en los demás países del estudio. Las cifras de un 53\% en la antigua Alemania del Este y de un $30 \%$ en el resto del país revelan, sin duda, una reducción drástica del sector público local, si bien es cierto que muchos empleado públicos fueron trasladados del núcleo duro de la administración municipal a empresas privadas participadas mayoritariamente por los Ayuntamientos.

En cuanto a España, las cifras son muy diferentes y denotan los profundos cambios en sus sistema intergubernamental en el que se ha producido una drástica reducción de personal en el nivel central que se ha correspondido con un incremento considerable del personal al 
Tabla 6. Variación del Empleo Público

\begin{tabular}{|c||c|c|c|c|c|}
\hline & Período & Estatal & Regional & Local & $\begin{array}{c}\text { Total Empleo } \\
\text { Público }\end{array}$ \\
\hline Alemania & $1991 / 2004$ & $\begin{array}{c}-24 \% \\
\text { (Bund) }\end{array}$ & $\begin{array}{c}-18 \% \\
\text { (Länder) }\end{array}$ & $\begin{array}{c}\text { Antiguos Länder: } \\
-30,0 \% \\
\text { Nuevos Länder } \\
-53,3 \%\end{array}$ & $-23 \%$ \\
\hline Francia & $1994 / 2003$ & $+7 \%$ & & $+24,0 \%$ & $+13 \%$ \\
\hline Reino Unido & $1990 / 2003$ & $-36 \%$ & & $-5 \%$ & $-4 \%$ \\
\hline Suecia & $1996 / 2003$ & $-3 \%$ & & $+0,5 \%$ & $-2 \%$ \\
\hline Italia & $1992 / 2004$ & $-11 \%$ & & $-16 \%$ & $-7 \%$ \\
\hline España & $1995 / 2005$ & $-41 \%$ & $\begin{array}{c}\text { Autónomas } \\
+92 \%)\end{array}$ & $+15 \%$ & $+13 \%$ \\
\hline
\end{tabular}

Fuente: Elaboración propia a partir de datos proporcionados por Dexia (2008)

servicio de las Comunidades Autónomas y, en menor medida un aumento de las plantillas en los gobiernos locales. Por sí sólo, este dato pone de manifiesto el alto grado de descentralización operado en España.

\section{EL GASTO PÚBLICO LOCAL}

Es evidente que el estatus de los gobiernos locales y su grado real de autonomía en el marco del sistema intergubernamental depende, de modo decisivo, de su posición financiera, medida tanto en términos de su porcentaje en relación al total de ingresos públicos como en porcentaje de ingresos propios y transferencias de otras administraciones, datos que se ilustran en el cuadro 5.

Tabla 5

\begin{tabular}{|c|c|c|c|}
\hline & $\begin{array}{c}\text { Porcentaje de ingresos de los } \\
\text { municipios respecto al total } \\
\text { de ingresos públicos. }\end{array}$ & $\begin{array}{c}\text { Porcentaje de ingresos } \\
\text {,propios“ respecto al } \\
\text { total de ingresos de los } \\
\text { municipios }\end{array}$ & $\begin{array}{c}\text { Porcentaje de las } \\
\text { transferencias respecto al } \\
\text { total de ingresos de los } \\
\text { municipios }\end{array}$ \\
\hline Alemania & $12 \%$ & $27 \%$ & $31,9 \%$ \\
\hline Francia & $8,6 \%$ & $52,0 \%$ & $31,5 \%$ \\
\hline Reino Unido & $\begin{array}{c}14,0 \% \\
\text { (los dos niveles) }\end{array}$ & $15,4 \%$ & $\begin{array}{c}48,2 \% \\
\text { los dos niveles) }\end{array}$ \\
\hline Suecia & $26,7 \%$ & $68,5 \%$ & $21,6 \%$ \\
\hline Italia & $9,4 \%$ & $34,2 \%$ & $33,7 \%$ \\
\hline España & $11,7 \%$ & $30 \%$ & $36,2 \%$ \\
\hline
\end{tabular}

Fuente: Elaboración propia a partir de datos proporcionados por Dexia (2008)

En dicho cuadro cabe destacar el alto porcentaje de recursos financieros (cerca del $27 \%$ del total de gasto público) gestionados por los municipios suecos, lo que subraya su importancia funcional. España y Alemania se encuentran en una posición intermedia aunque 
no muy alejados de Francia e Italia en el que la participación de los municipios en el gasto es menor y por debajo del 10\%. Los datos del Reino Unido no son comparables al no estar disponible más que su agregado en varios niveles (districts/boroughs, counties/ unitary authorities).

Por cuanto, como se observa, la estimación de la autonomía financiera y presupuestaria del gobierno local puede realizarse a partir de dos indicadores. Primero, el porcentaje de los tributos propios respecto a los ingresos totales, entendiendo por tributos propios aquellos que pueden ser establecidos por el Pleno en todos sus términos. Están, por otra parte, los tributos que los municipios "comparten" (revenue sharing) con otros niveles de gobierno en cuyo caso la estructura del impuesto se regula por el legislador central o federal, incluyendo el porcentaje de los recursos que se atribuyen al nivel local, de manera que, respecto a tales ingresos "compartidos" los municipios dependen de las decisiones políticas del nivel superior.

Desde esta perspectiva se destaca la autonomía de los municipios suecos en los que la casi totalidad de sus ingresos provienen de sus tributos propios y algo similar sucede en el gobierno local francés. En el caso de Alemania y España sólo un tercio de sus ingresos tributarios provienen de los impuestos propios y en Inglaterra el porcentaje es mucho menor, con un margen de maniobra presupuestario muy reducido a partir de la centralización impulsada por el gobierno central desde los años ochenta. Del mismo modo, el grado de autonomía financiera de los municipios se revela también a partir de su dependencia de las transferencias estatales que en los municipios suecos es menor (20\%) mientras que en Alemania, Francia, Italia y España está entre el 30 y el 36\% y en el Reino Unido asciende, para todos los niveles locales, al $48 \%$.

A la luz de lo que se termina de decir tenemos que, por un lado, los recursos financieros de los gobiernos locales son, en general, limitados y, por otro, que existen diferencias notables entre los países considerados en cuanto a su autonomía respecto a los demás niveles territoriales. Pero el punto central en lo que toca a la situación financiera tiene que ver con las restricciones presupuestarias derivadas de los ajustes fiscales que requiere el hacer frente a las crisis económicas. Los municipios se ven abocados a atender cada vez más demandas de asistencia y servicios sociales $y$, en muchos casos hacer frente a competencias transferidas sin los correspondientes recursos materiales, lo que provoca su ahogo financiero. A este respecto y en el caso de Alemania, se ha introducido tanto en la Constitución como en los Estatutos de los Länder una cláusula (Konnexitätsprinzip) según la cual sólo podrán ser transferidas competencias a los municipios si se acompañan de una transferencia equivalente de recursos, aunque en la práctica se está viendo como los niveles superiores se valen de argucias procedimentales para evitar dicha transferencia.

\section{CONCLUSIONES.}

El análisis comparativo de la evolución de los gobiernos locales revela la existencia de ciertas ambigüedades. Por una parte, se ponen de manifiesto los diversos factores que influencian las trayectorias institucionales. Así, las herencias y dependencias del sendero específicas de cada país dan lugar a continuidades e inercias en el desarrollo de los gobiernos locales. De otra parte, el análisis comparativo pone también de manifiesto que la existencia de factores dinamizadores que impulsan y fomentan los cambios. Entre ellos se encuentran 
las iniciativas políticas para descentralizar la arquitectura intergubernamental, la consolidación de la estructura territorial con el fortalecimiento de los municipios, la profundización en el potencial democrático y participativo de los ciudadanos y el robustecimiento del liderazgo político y administrativo en el sistema local, así como la modernización de su aparato administrativo.

Con todo, se observan tendencias que, dependiendo de las condiciones específicas de cada país, manifiestan tanto semejanzas como diferencias.

En general, se percibe un movimiento hacia la descentralización. En España como resultado del cambio de régimen político a partir de 1978; en Italia como consecuencia de la crisis constitucional y política de los años 90 han emergido y se están consolidando tendencias cuasifederales. Por el contrario, en Reino Unido Escocia y Gales se han visto "regionalizados" mientras que en Inglaterra las regiones han pasado a ocuparse de asuntos técnicos produciéndose un proceso de centralización iniciado por el gobierno tatcherista y al que se adhirió también el actual gobierno laborista.

En cuanto a la estructura territorial del nivel local persisten las diferencias entre países del modelo norte-europeo (Reino Unido, Suecia, algunos Länder alemanes con municipios de gran tamaño sin intermunicipalidades) y del modelo sur-europeo (Francia, España e Italia con pequeños municipios y con entes intermunicipales). Sin embargo, recientemente se observan tendencias hacia una "consolidación" de la estructura territorial mediante la creación de municipios mayores e integrados al tiempo que se abolen los entes intermunicipales. Este fenómeno es observable tanto en algunos Länder (particularmente en la antigua Alemania del Este) como en las recomendaciones (aun no implantadas) que se contienen el informe del Comité Balladur en Francia.

Hay que tener presente, empero, que no obstante las diferencias existentes en cuanto al ejercicio de competencias (sobre todo en los ámbitos de educación y salud), se observan rasgos comunes en cuanto que, por un lado, respecto a determinadas responsabilidades tradicionales los municipios están perdiendo funciones debido a la transferencia (outsourcing) o privatización a favor de agentes y prestadores privados, semi-públicos y públicos. En este respecto, resulta ejemplar la esfera del abastecimiento de servicios esenciales. Por otro lado, las competencias del nivel local se están ampliando como resultado de las transferencias y delegaciones de otras administraciones. Esto ha sido impulsado a raíz de las crisis económicas de carácter cíclico que en algunos países van acompañadas de incrementos significativos en las cifras de desempleo y, por ello, de las exigencias, cada vez más urgentes a los niveles locales para involucrarse en proyectos e iniciativas locales para combatir el desempleo y sus consecuencias sociales. Aunque no deja de ser significativo, en esta evolución, la brecha progresiva entre gastos crecientes e ingresos menguantes.

El impulso por fortalecer el potencial democrático y participativo se observa en todos países. Aunque, de hecho, sólo en Alemania e Italia se han implantado procedimientos de refrenda decisorios (con pleno poder de complementar y hasta, en casos reglados, suplir las potestades del Pleno) mientras que en Francia y en España tienen sólo un valor consultivo y, en ocasiones, se utilizan para algo alejado del ejercicio de competencias por parte de los municipios, como ocurre con las iniciativas llevadas a cabo en algunos municipios catalanes. $\mathrm{Ni}$ in Inglaterra ni in Suecia se ha introducido el mecanismo del referéndum lo que refleja el predominio del principio de la democracia representativa (que, en el Reino Unido, tiene su sustento en el principio de la parliamentary sovereignty). 
De modo semejante se observa una intención común de reformar la institucionalización del liderazgo político y administrativo. Pero, en este ámbito, persiste también una "dependencia de sendero" en el sentido de que, por un lado, los países de la tradición de "gobierno local por comités" (government by commission, es decir Inglaterra y Suecia) están llevando a cabo la reformas esencialmente en el marco de este modelo tradicional hacia una forma de "sistema parlamentario local". Por otro lado, entre los países de la Europa continental (con una tradición de reparto de competencias -y de poder- entre los Plenos y el alcalde/maire/sindaco/Bürgermeister), destacan Alemania e Italia en donde se introdujo la elección directa del Bürgermeister y del Sindaco, de manera que en estos países puede hablarse de un modelo de alcalde presidencialista. Y algo similar sucede en España y Francia donde la figura del Alcalde, elegido por el Pleno, está progresivamente adquiriendo perfiles presidencialistas.

Del mismo modo, se puede constatar en todos países un afán por reformar y modernizar la administración pública local. Sin duda, existen diferencias entre las distintas "familias" de países. Por una parte, en el Reino Unido (como ejemplo paradigmático del modelo anglosajón) el movimiento de la Nueva Gestión Publica (New Public Management), con su énfasis en la transferencia (outsourcing) y la introducción de criterios de mercado (marketisation) en la prestación de servicios públicos, ha transformado considerablemente la administración local, aproximándola a un funcionamiento similar al sector privado. Por el contrario, en los países continentales, la tradición del Estado de Derecho (Rechtsstaat) y el arraigo del modelo burocrático weberiano ha propiciado que los impactos de la Nueva Gestión Publica resulten muy limitados.

\section{BIBLIOGRAFÍA}

ALBA, C. (1997): “Gobierno Local y Ciencia Política: Una aproximación” en Alba, C. y. Vanaclocha, F. J. (eds.) El sistema político local: un nuevo escenario de gobierno, Madrid, Universidad Carlos III/BOE.

ALBA, C. y VANACLOCHA, F.J. (comps.): 1997: "Presentación", en El sistema político local: un nuevo escenario de gobierno, Madrid, Universidad Carlos III y Boletín Oficial del Estado, pp. 9-12.

BALDERSHEIM, H., CITRONI, G., MARCOU, G., McELDOWNEY, J. y WOLLMANN, H. (2010): "From public service to commodity. The de-municipalization (and re-municipalization?) of energy provision in Germany, Italy, France, the U.K. and Norway", en: Wollmann, H. y Marcou, G. (eds.), The delivery of social/public service in Europe. Between State, local government and market, Aldershot, Edward Elgar (en prensa).

BRUGUÉ, Q. y GOMÁ, R. (1998): “Gobierno local: de la nacionalización al localismo y de la gerencialización a la repolitización” en Brugué, Q. y Gomá, R. (comps.), Gobiernos locales y políticas públicas : bienestar social, promoción económica y territorio, Barcelona, Ariel, pp. 15-35.

COMITÉ BALLADUR. (2009): "Il est temps de décider", en línea : http://www.francematin.info/L e-rapport-du-comite-Balladur-a-telecharger-en-exclusivite_a18303.html (consulta 15 diciembre 2009)

DEXIA (2006): Les Fonctions Publiques Locales, Paris, CNPFT.

DEXIA (2008): Subnational Governments in the European Union, Paris, CNPFT.

GROTZ, F. (2000): Politische Institutionen und post-sozialistische Parteiensysteme in Ostmitteleuropa, Opladen, Leske \& Budrich.

HALL, P.A. (1993): "Policy Paradigms, Social Learning and the State", Comparative Politics, 25, pp. 275-296. 
HALL P. y TAYLOR, R., (1996): "Political Science and the Three New Institutionalismus", Political Studies, 44/5, pp: 936-957.

KATO, J. (1996): "Institutions and Rationality in Politics. Three Varieties of Neo-Institutionalists", British Journal of Political Science, 26, pp. 553-582.

KERSTING, N., CAULFIELD, J. ANDREW, N. y WOLLMANN, H. (2009: Local Government Reform in Global Perspective, Wiesbaden, VS Verlag.

KOOIMAN, J. (1993): Modern governance: new government-society interactions, Londres, Sage.

KUHLMANN, S. (2009): "Une convergence des modèles administratives locaux? Etude comparé de la décentralisation en France et en Allemagne", Pouvoirs Locaux, 81 (II/2009), pp. 81-88

KUHLMANN, S., BOGUMIL, J. y GROHS, S. (2008): "Evaluating administrative modernization in German local governments: success or failure of the "New Steering Model"?" Public Administration Review; 68, (5), pp. 851-863.

MARCH, J.G. y OLSEN J.P., (1984): "The new institutionalism: organizational factors in political life", American Political Science Review, 78, pp. 738-749

- (1989): Rediscovering Institutions, Nueva York, Free Press.

MARCOU, G., y WOLLMANN, H., (2008): "Descentralización y democracia local en el mundo: Europa” Gold Report, Ciudades y Gobiernos Locales Gold, Barcelona, pp. 197-233, en línea: http://ww w.cities-localgovernments.org/gold/Upload/gold_repo rt/05_europe_es.pdf (consulta: 9 enero 2010)

MOE, T.M. (2005): "Power and Political Institutions", Perspectives on Politics, vol 3,2, pp. 215-233.

NOHLEN, D. (2006): El Institucionalismo contextualizado. La relevancia del contexto en el análisis y diseño institucionales, México, D.F., Porrúa-UNAM.

NORTH, D. (1992): Institutionen, institutioneller Wandel und Wirschasftleistung, Tübingen, Mohr.

NORTON, A. (1994): International Handbook of Local and Regional Government. Aldershot, Edward Elgar.

PETERS, G. (1996): “Institutionalism old and new”, en Goodin, R.E. y Klingenmann, H.D. (eds.), A New Handbook of Political Science, Oxford, Oxford University Press

- (1999): Institutional Theory in Political Science: The New Institutionalism, Londres, Routledge.

- (2003): El Nuevo institucionalismo. Teoría institucional en ciencia política, Barcelona, Gedisa.

POLLITT, CH. y BOUCKAERT, G. (2004): Public Management Reforms, Oxford, Oxford University Press,

SKOCPOL, T. (1985): "Bringing the State Back In: Strategies of Analysis in Current Research", en Evans, P.; Rueschemeyer, D. y Skocpol, T. (eds.), Bringiong the State Back In, New York, Cambridge University Press.

THELEN, K., (1999): "Historical Institutionalism in Comparative Politics", Annual Review of Political Science, 199/2, pp. 369-404.

TRUE, J.L., JONES B.D. y BAUMGARTNER, F.R. (1999): "Punctuated-equilibrium Theory: Explaining Stability and Change in American Policymaking" en Sabater, P.A. (ed.), Theories of the policy process, Oxford, Westview Press.

WOLLMANN, H. (2004): "Local government reforms in Great Britain, Sweden, Germany and France: between multi-function and single-purpose organisations", in: Local Government Studies, special issue, vol. 30, no.4, pp. 639-668.

- (2007): "Changes, Ruptures, and Continuities in European Local Government Systems. Between Government and Governance", in: Lazin, F.; Evans, M.; Hoffmann-Martinot, V.; Wollmann, H. y Bouckaert, G. (eds.) (2006), Local Government Reforms in Countries in Transition, Lanham, Rowman \& Littlefield Publishers, pp. 15-38.

- (2008a): Reformen in Kommunalpolitik und -verwaltung. England, Schweden, Frankreich und Deutschland im Vergleich, (hrsg. Wüstenrot-Stiftung), VS Verlag, Wiesbaden.

- (2008b): Comparing Local Government Reforms in England, Sweden, France and Germany (ed. Wüstenrot Stiftung), en: www.wuestenrot-stiftung.de/download/local-government

- (2008c): "Reforming local leadership and local democracy. The cases of England, Sweden, Germany and France in comparative perspective", in: Local Government Studies, vol. 34, no. 2, pp. 279-298. 
- (2009a): "Des systèmes communaux européens en mutation: Etude comparée de la France, de l'Allemagne, du Royaume Uni, de l'Italie et de la Suède", en: Pouvoirs Locaux, no. 81 (II/2009), pp. 57-80.

- (2009b): "Local level territorial reforms in East German Länder", in Swianiewicz", P. (ed.), Territorial consolidation - Lessons Learned? Budapest, Open Society Institute, LGI series.

\section{Breve currículo:}

\section{Hellmut Wollmann}

Catedrático emérito de políticas públicas y administración pública en la Universidad Humboldt de Berlín. En estos ámbitos ha llevado a cabo numerosos proyectos de investigación cuyo objeto de estudio ha sido el análisis comparativo a nivel europeo de los gobiernos subnacionales y locales y que han dado lugar a un buen número de publicaciones científicas. Entre ellas, su reciente libro aparecido en 2010, del que es coeditor junto con Gerard Marcou, The provision of public services in Europe de la editorial Edward Elgar.

\section{Ángel Iglesias Alonso}

Doctor en Ciencia Política y de la Administración y Profesor Titular de Ciencia Política en la Universidad Rey Juan Carlos. Es profesor visitante de diversas universidades extranjeras entre las que se encuentran el Instituto de Estudios Políticos de París y la Universidad Alemana de Ciencia de la Administración en Speyer. Participa en varios proyectos de investigación nacionales e internacionales que tienen como objeto de estudio los gobiernos locales y en este ámbito es Premio Nacional de Gobierno y Administración Local por el Instituto Nacional de Administración Pública y Premio Fernando Albi de Gobierno Local por la Diputación de Alicante. 